\title{
Contact-Shifted NMR of Spinach Ferredoxin: Additional Resonances and Partial Assignments
}

\author{
I. SALMEEN \\ Scientific Research Staff, Ford Motor Company, Dearborn, Mighigan 48120
}

G. PALMER ${ }^{1}$

Department of Biological Chemistry and Biophysics Research Division, Institute of Science and Technology University of Michigan, Ann Arbor, Michigan 48105

Received February 14, 1972; accepted March 24, 1972

\begin{abstract}
We have extended the study of the contact-shifted resonances in the nmr spectra of oxidized and reduced spinach ferredoxin to lower magnetic field than reported by others. We have found two resonances not observed previously: one at $37 \mathrm{ppm}$, corresponding to as many as eight protons, in the oxidized protein; and the other at $43 \mathrm{ppm}$, corresponding to two protons, in the reduced protein. Assignments are proposed for these resonance and for those previously reported. We conclude that amino acid residues other than eysteine may participate in coordinating the iron atoms. By comparing the line widths in our $60 \mathrm{MHz}$ spectra with those in the published $220 \mathrm{MHz}$ spectra, by evaluating hyperfine coupling constants, and by an independent measurement of the electron paramagnetic resonance line width at $230^{\circ} \mathrm{K}$, we estimate the electronic spin-relaxation time of the reduced protein to be $c a .1 \times 10^{-11}$ sec. With this value for $\tau$ we determine the dipolar contribution to the line width and hence estimate the distances between the contact-shifted protons and the paramagnetic center.
\end{abstract}

The active center of the two-iron ironsulfur proteins is believed to be a binuclear cluster comprising both iron atoms each of which is tetrahedrally coordinated by four sulfur atoms. Two of these sulfur atomsthe labile sulfides - are bridging ligands common to both iron atoms, the other four sulfur atoms, external to the binuclear center, are provided by cysteine residues $(1,2)$. In the oxidized state both iron atoms are highspin ferric, in the reduced state one remains high-spin ferric and the other converted to the high-spin ferrous state $(2,3)$. These iron atoms interact via a moderately strong antiferromagnetic exchange interaction which is decreased by approximately $50 \%$ on reduction (4).

The $\mathrm{nmr}$ spectrum of spinach ferredoxin

1 Supported by NIH Grant GM-12176. in both redox states exhibits resonances to low-field of the diamagnetic proton region $(0-10 \mathrm{ppm})$ and these have been assigned as the $\beta-\mathrm{CH}_{2}$ protons of the liganding cysteines (5) which are "contact-shifted" to their observed positions by virtue of the paramagnetism of the metal ions (cf. 6). An unexplained feature of that work was the observation that these resonances could be grouped into two classes, those for which the position shifted to lower field with increasing temperature and those that shifted to higher field.

A consequence (2) of the above model is that protons which "sense" the spin density on the ferrous ion will show a positive temperature dependence, while those that "sense" the ferric ion will show the observed negative temperature dependence, and a 
detailed semiquantitative prediction of line positions has been made (2). In addition it was noted that only one contact-shifted proton was observed in the $\mathrm{nmr}$ spectrum of the oxidized protein, whereas a maximum of eight $\beta-\mathrm{CH}_{2}$ protons was to be expected.

For this reason we have searched for additional nmr lines to the limits of the instrumentation available to us and have observed resonances in both redox forms of the protein which are consistent with the predictions of Dunham et al. (2) and help to clarify the assignments arising from their analysis.

\section{METHODS AND RESULTS}

Proton magnetic resonance (pmr) data were taken at $60 \mathrm{MHz}$ using a Brüker HFX -60 spectrometer equipped with $12 \mathrm{kHz}$ audio modulation, ${ }^{19}$ fluorine field-frequency lock, $10-\mathrm{mm}$ spinning sample tubes, and Brüker variable temperature controller and Dewar inserts. Hexafluorobenzene, used for the lock signal, was contained in a $5-\mathrm{mm}$ sample lube inserted inside the 10 -mm sample tube and rigidly positioned with Teflon plugs bored to tightly accommodate the $5-\mathrm{mm}$ tube. The frequency-swept spectrum was referenced to $1 \%$ DSS dissolved in the sample. Either a Varian C1024 computer of average transients or a Digital Equipment Corporation PDP8I computer was used for time averaging. The integrated intensity of the resonance in the oxidized protein was estimated by cutting out the recorded spectrum and weighing the paper against the weight of the spectrum from the known concentration of DSS dissolved in the solution. This procedure was necessitated by the extreme width of the resonance and by the fact that at the high sensitivity required to observe this resonance, contributions from small amounts of dispersion mode signal from upfield resonances made baseline determination very difficult for electronic integration. This estimate of integrated intensity probably is in error by as much as $\pm 30 \%$.

Spinach ferredoxin was prepared according to the method of Petering and Palmer (7). Prior to use, the protein was dialyzed overnight against a solution of $0.01 \mathrm{M} \mathrm{NaCl}$ and $0.01 \mathrm{M}$ potassium phosphate buffer $\mathrm{pH} 7.4$ at $4^{\circ} \mathrm{C}$, and stirred with a continuous stream of nitrogen bubbled through the dialysis solution. The sample was then exchanged into $99.98 \% \mathrm{D}_{2} \mathrm{O}$ via two cyeles of freeze-drying and finally dissolved in a volume of $\mathrm{D}_{2} \mathrm{O}$ sufficient to give final concentration of $c a .20 \mathrm{mM}$. The samples were reduced in the nmr tubes by adding 0.1 $\mathrm{ml} 0.5 \mathrm{~m}$ sodium dithionite dissolved in $1 \mathrm{~m}$ potassium phosphate, $\mathrm{pH} 7.3$, which had been prepared in $\mathrm{D}_{2} \mathrm{O}$. The nmr sample tube was flushed thoroughly with nitrogen before reduction. The solution of dithionite was introduced either by in. jecting with a hypodermic syringe through a selfsealing serum cap used to stopper the top of the nmr tube, or else by adding the solution while constantly flushing with nitrogen and then quickly

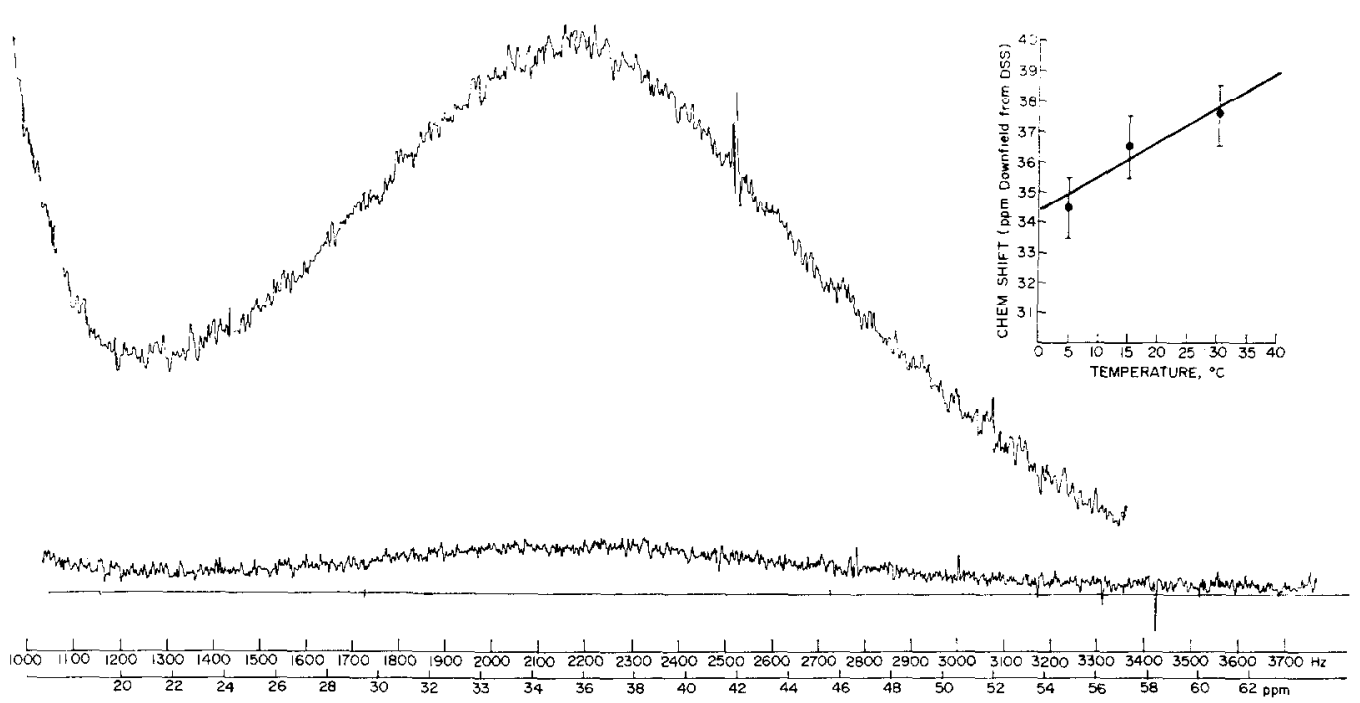

Fig. 1. Low field $60 \mathrm{MHz}$ spectrum, oxidized spinach ferredoxin. Lower trace single scan. Upper trace sum of 16 scans. Vertical scales not commensurate. Note unconventional display with magnetic field decreasing to the right. 


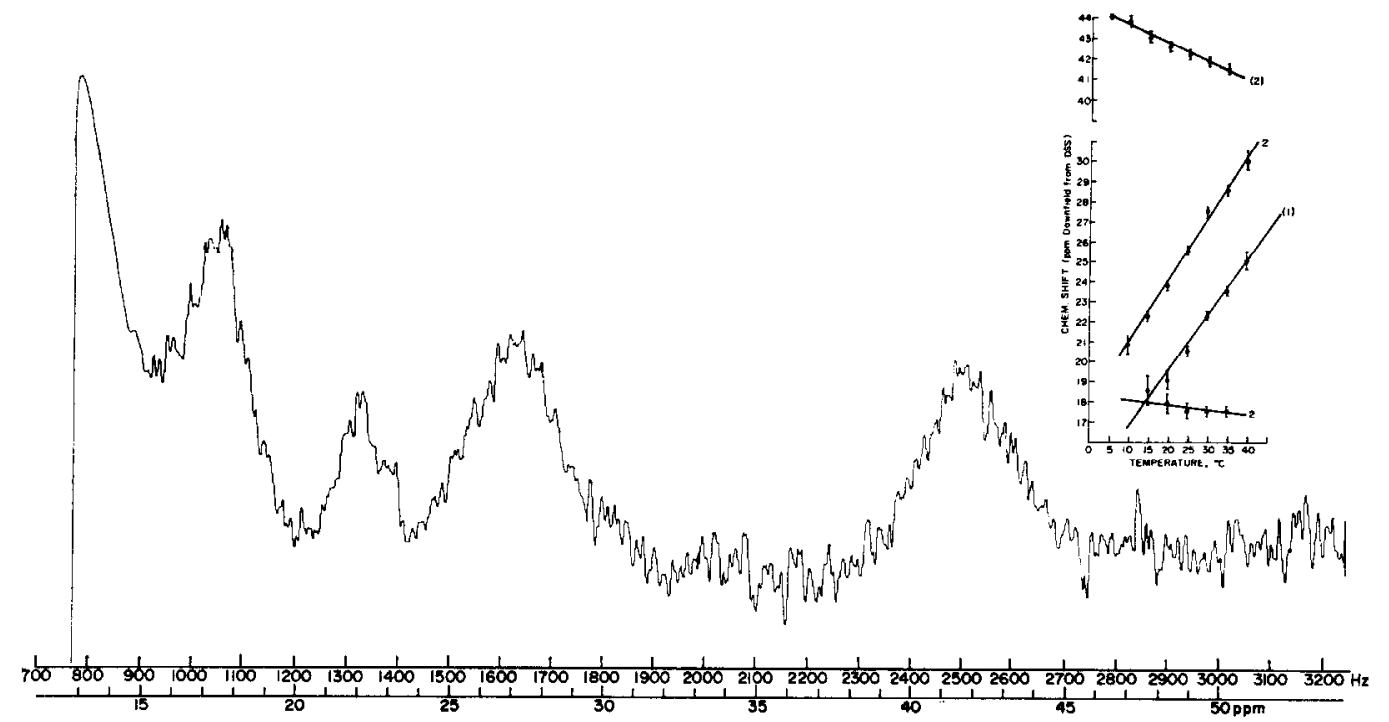

Frg. 2. Low field $60 \mathrm{MHz}$ spectrum, reduced spinach ferredoxin. Sum of 16 passes. Resonance at $42 \mathrm{ppm}$ not reported previously. Number of protons corresponding to each resonance indicated by numbers adjacent to plots of temperature dependences. Note unconventional display as in Fig. 1.

stoppering the tube with a serum cap. In either case, the sample had not reoxidized after $12 \mathrm{hr}$.

Figure 1 is the $60-\mathrm{MHz}$ spectrum of oxidized spinach ferredoxin. At this lower frequency the resonance observed by Poe et al. (5) at $15 \mathrm{ppm}$ appears as a shoulder on the tail of the non-contactshifted resonances. The broad resonance centered at $c a .36 \mathrm{ppm}$ was not observed previously. We estimate that it contains $6 \pm 2$ protons; its temperature dependence is shown in the inset. (Note that the spectrum is displayed unconventionally; magnetic field is decreasing to the right.)

Figure 2 shows the spectrum of the reduced protein; no resonances were observed in the range 55-72 ppm. The temperature dependence and relative intensity of these lines are given in the inset. The resonance in the region of $42 \mathrm{ppm}$ was not observed previously while the remaining lines are among those reported by Poe et al. (5).

\section{DISCUSSION}

At this time we are unable to identify uniquivocally the protons responsible for the observed contact-shifted nmr resonances. Nevertheless, several conclusions can be drawn from the available data and we wish to present the arguments which lead to these conclusions. To facilitate comparison with the published spectra of Poe et al. (5) all resonances will be designated by their shifts in $\mathrm{ppm}$ at $5^{\circ} \mathrm{C}$.
In attempting to analyze these spectra we have relied on the semiquantitative model of Dunham et al. (2). In this model the Hamiltonian describing the contact interaction in the reduced protein has the form:

$$
\mathfrak{H}=\sum_{i=1}^{M} a_{i}\left\langle S_{z_{1}}\right\rangle I_{z_{i}}+\sum_{j=1}^{N} a_{j}\left\langle S_{z_{2}}\right\rangle I_{z_{j}}
$$

where the subscripts 1 and 2 refer to the ferric and ferrous ions, respectively, and the summations are over the $i(j)$ protons. For the oxidized protein $\left\langle\boldsymbol{S}_{z_{1}}\right\rangle=\left\langle\boldsymbol{S}_{z_{2}}\right\rangle$ and this Hamiltonian requires just a single term. The expectation values $\left\langle S_{z_{i}}\right\rangle$ include the weighted averages of all thermally accessible states. As a result of this thermal averaging, protons in the reduced protein which are contact-shifted by interaction with the ferric ion move upfield (i.e., toward DSS) with increasing temperature and protons which are contact-shifted by interaction with the ferrous ion move downfield with increasing temperature. The parameters required to quantitatively determine these expectation values as a function of temperature were calculated by Dunham et al. (2) by fitting the temperature dependence of magnetic susceptibility and Mössbauer data.

In addition to relying upon the conclusions 
from this model, we further assume that a $\mathrm{C}-\mathrm{C}$ single bond attenuates the transmission of spin density by about an order of magnitude; a factor of 11-15 was found by Eaton et al. (8) in alkyl-substituted nickel (II) aminotroponeiminates. Thus, for cysteine bonded to iron via the sulfur atom, the $\beta-\mathrm{CH}_{2}$ protons should be shifted about 10 times further than the $\alpha$-CH proton. (This assumption requires that the only contribution to the contact-shift is transmission of spin-density through chemical bonds. The rather small anisotropy of the $g$-tensor in reduced spinach ferredoxin implies that the pseudocontact terms are negligible and that this assumption is reasonable.)

We assign the broad resonance at ca. 34 ppm $\left(5^{\circ} \mathrm{C}\right)$ in the oxidized protein as due to the eight $B-\mathrm{CH}_{2}$ protons of the four cysteine residues which are presumed to function as ligands to the ferric ions. This assignment is based solely on the observation that the intensity of this resonance accounts for approximately eight protons.

Accepting this assignment, we would expect to find the four $\alpha-\mathrm{CH}$ protons of these cysteine residues in the range of 6-7 ppm downfield from DSS, ${ }^{2}$ and would thus be obscured by the large number of resonances which occur in this region. However, the data of Poe et al. (5) on oxidized spinach ferredoxin taken at 5 and $30^{\circ} \mathrm{C}$ are significantly different at the two temperatures in several regions including that around $7 \mathrm{ppm}$. Furthermore their data on oxidized parsley ferredoxin shows a resonance in the region of $6.3 \mathrm{ppm}$ with a temperature dependence strongly suggestive of a contact-shifted resonance.

As a result of these assignments, we conclude that the contact-shifted resonance at 14.7 ppm, reported by Poe et al. (5) corresponding to a single proton, is not due to a cysteine proton, but to some other amino acid residue. If this is true, these are the first data to indicate that amino acids other than cysteine have a role in coordinating the metal ions.

These conclusions regarding the oxidized

2 The temperature-independent diamagnetic chemical shift for the $\alpha-\mathrm{CH}$ protons is taken as 4 ppm, and for the $\beta-\mathrm{CH}_{2}$ protons as $3 \mathrm{ppm}(9)$. protein can be used to provide a reasonable explanation of the spectrum of the reduced protein. We make two additional assumptions:

(1). No change in coordination geometry of the binuclear center accompanies reduction so that the hyperfine interaction constants of the ferric and ferrous protons are unchanged on reduction. ${ }^{3}$ When the values of the hyperfine constants remain unchanged, the positions of the proton resonances in the reduced protein are given by:

$$
\nu_{\text {red }}=\nu_{\text {ox }}\left\langle S_{z}\right\rangle_{\mathrm{red}} /\left\langle S_{z}\right\rangle_{\mathrm{ox}} .
$$

(2). The resonances at $13-21 \mathrm{ppm}$ which account for four protons and which exhibit the strong negative temperature dependence anticipated for ferrous contact-shifted protons are the $\beta-\mathrm{CH}_{2}$ cysteine protons. With $\left\langle S_{z_{1}}\right\rangle_{o x}=1.7 \times 10^{-3}$, (2) then $\left\langle S_{z_{2}}\right\rangle_{\text {red }}=$ $0.8 \times 10^{-3}$ which in turn establishes $\left\langle S_{z_{1}}\right\rangle_{\text {red }}=24 \times 10^{-3}$, corresponding to a value for $J$ of about $65 \mathrm{~cm}^{-1}$. [Strictly speaking we only require that the total unpaired spin density at the ferrous protons be decreased by about one-half; it seems likely that a small fraction of this spin density originates at the ferric ion (2).]

We thus expect that the position of the $\beta-\mathrm{CH}_{2}$ cysteinyl protons of the ferric ion in the reduced protcin should be about 468 ppm. This is well beyond the limits of the frequency sweep of our spectrometer and, furthermore, observation of this resonance

\footnotetext{
${ }^{3}$ Unfortunately, the available spectroscopic data are not very helpful in evaluating this assumption. The observation that the quadrupole splitting and isomer shift of the ferric ion in the Mössbauer spectrum of reduced ferredoxin is identical with that in the oxidized protein is of little significance because the experimental values are those which characteristically are insensitive to changes in the geometry of the ligands coordinating the iron.

The assumption that the values of the hyperfine constants for the protons of the ferrous ion are unchanged on reduction is predicated on the conclusion from Mössbauer spectroscopy that the extra ferrous electron resides in a $d_{z^{2}}$ orbital. The ferrous ion is believed to be tetrahedrally coordinated and in this geometry $d_{z^{2}}$ is only weakly bonding. Consequently the extra electron should have a small effect on the hyperfine constants.
} 
would be difficult because the line width (see below) is proportional to $\left\langle S_{1}^{2}\right\rangle$ which is ca. 20 times larger in the oxidized protein than in the reduced.

With a factor of 10 attenuation across a $\mathrm{C}-\mathrm{C}$ single bond, we then expect to find the two $\alpha-\mathrm{CH}$ cysteinyl protons associated with the ferric ion at about $47 \mathrm{ppm}$. This is suggestively close to the position of the line which is observed at $44 \mathrm{ppm}\left(5^{\circ} \mathrm{C}\right)$, with an intensity corresponding to two protons and which has a temperature dependence indicating interaction with the ferric ion. We therefore assign this line as due to the two $\alpha-\mathrm{CH}$ ferric cysteines. In the same vein it follows that the two $\alpha$ protons of the ferrous cysteines be at about $4-5 \mathrm{ppm}$.

The two resonances at 10 and $18 \mathrm{ppm}$ in the reduced protein, each of unit intensity, and with a temperature dependence which corresponds to ferric ion, are apparently not due to cysteine protons, nor do they appear to be related to the resonance at $15 \mathrm{ppm}$ in the oxidized protein. Again we must conclude that these arise from amino acid residues not previously anticipated to be in the coordination sphere of the metal ions.

Once these assignments are accepted, the data suggest that the line widths can be interpreted to provide estimates of the distances between the paramagnetic center and the various contact-shifted protons. In general, the line widths are given by the Solomon-Bloembergen equations (10):

$$
\begin{aligned}
& \frac{1}{T_{2_{j}}}=\frac{1}{15}\left\langle S_{i}{ }^{2}\right\rangle \frac{\mathrm{g}_{e}{ }^{2} \beta_{e}{ }^{2} \mathbf{g}_{n}{ }^{2} \beta_{n}{ }^{2}}{r^{6}}\left[7 \tau_{e}+\frac{13 \tau_{e}}{1+\omega_{S}{ }^{2} \tau_{e}{ }^{2}}\right] \\
& +\frac{1}{3}\left(\frac{A_{j}}{\hbar}\right)^{2}\left\langle{S_{i}}^{2}\right\rangle\left[\tau_{e}+\frac{\tau_{e}}{1+\omega_{s}{ }^{2} \tau_{e}{ }^{2}}\right],
\end{aligned}
$$

where $\tau_{\varepsilon}$ is the electronic spin-lattice relaxation time, $\omega_{S}$ is the electron Larmor frequency, and $\left\langle S_{i}{ }^{2}\right\rangle=S(S+1)\left(\mathbf{S}_{i} \cdot \mathbf{S}\right.$ $(S)^{2}$ thermally averaged over all states. This expression assumes that the electronic relaxation time is much shorter than the rotational correlation time.

Three general arguments suggest that either $\tau_{e} \approx 1-3 \times 10^{-11} \mathrm{sec}$ or $\tau_{e}<1 \times 10^{-13}$ sec. (1)From the observed line positions and the values for $\left\langle S_{z_{i}}\right\rangle$, the isotropic hyperfine coupling constants can be determined. ${ }^{4}$ Knowing these coupling constants, a value for $\tau_{e}$ can be computed for each contactshifted resonance which would be sufficient for the isotropic term alone to account for the observed line width, assuming that the dipolar contribution is negligible and $\omega_{S} \tau_{e} \gg$ 1. These values are summarized in Table I as $\tau_{h j}$. The actual value for $\tau_{e}$ cannot be longer than the shortest of these values. For the line corresponding to this shortest value of $\tau_{h f}$, the isotropic hyperfine interaction alone accounts for the experimental line width, and any longer value for $\tau_{e}$ would predict a line width greater than that experimentally observed. Thus $\tau_{e}<3 \times$ $10^{-11}$. (2)To within the accuracy of estimating line widths $(c a . \pm 15 \mathrm{~Hz})$ from the published data of Poe et al. (5), the line widths appear to be identical at $220 \mathrm{MHz}$ and $60 \mathrm{MHz}$. This means that $\tau_{e}$ cannot be in the range $1 \times 10^{-11}$ to $4 \times 10^{-13} \mathrm{sec}$; if it were, then $\omega_{s} \tau_{c} \approx 1$ and we should be able to observe a field dependence for the line width $^{5}$. A one-to-one correspondence of all

${ }_{4}$ The observed line positions are given by

$$
\nu_{\mathrm{obs}}=A_{i}\left\langle S_{z}\right\rangle_{1,2}+\delta_{i}
$$

where the $\delta_{i}$ are the diamagnetic chemical shifts. In the temperature range of these measurements $\left\langle S_{z}\right\rangle_{1,2}$ are approximately linear functions of temperature; thus, the slopes of the observed temperature dependences are proportional to the slopes of $\left\langle S_{z}\right\rangle_{1,2}$, where the proportionality constants are the hyperfine coupling constants. In principle the hyperfine constants should be determined from the slopes whereupon the diamagnetic shift can be determined from the line position. Assignments should be based on these diamagnetic shifts. However, because of the experimental uncertainty in the slopes we ean only conclude that the diamagnetic shifts of the various protons are in the range 0-8 ppm. Therefore, for those resonances which we have attributed to cysteine, we determine the hyperfine constants from the line positions assuming a diamagnetic contribution of 4 and 3 ppm for the $\alpha$ and $\beta$ protons, respectively (9).

For the resonances at 11.2 and $18.0 \mathrm{ppm}$ in the reduced protein, and $14.7 \mathrm{ppm}$ in the oxidized, we can only place limits on the hyperfine constants because of the uncertainty in the diamagnetic contribution. For the various amino acids values between 2.0 and $8.0 \mathrm{ppm}$ are possible (9).

${ }^{5}$ We assume that $\tau_{e}$ is independent of field in 


\section{TABLE I}

\section{Values for Electron-Proton Separation in Reduced Protein Calculated from} Dipolar Contribution to the Line Widths

$(A / \hbar)$ is hyperfine interaction, $1 / T_{2 \text { obs }}$ is measured line width (half-width at half-maximum), $\tau_{\mathrm{bf}}$ is electronic relaxation time required to account for observed line width neglecting dipolar interaction, $r$ is the distance in angströms resulting from the evaluation of the dipolar contribution to the line widths.

\begin{tabular}{|c|c|c|c|c|c|c|}
\hline \multirow[b]{2}{*}{ Resonance $^{a}$} & \multirow[b]{2}{*}{$A / \mathbf{h}\left(\mathrm{rad} \cdot \sec ^{-1}\right)$} & \multirow{2}{*}{$\frac{1}{\left(\mathrm{rad} \cdot \mathrm{sec}^{-1}\right)}$} & \multirow[b]{2}{*}{$\tau_{h \cdot f}(\mathrm{sec})$} & \multicolumn{3}{|c|}{$r(\AA)^{c}$} \\
\hline & & & & $\begin{array}{c}\tau_{e}=2.5 \times \\
10^{-11}(\mathrm{sec})\end{array}$ & $\begin{array}{l}\tau_{e}=1 \times \\
10^{-11}(\mathrm{sec})\end{array}$ & $\begin{array}{l}\tau_{e}=4 X \\
10^{-13}(\mathrm{sec})\end{array}$ \\
\hline $11.2\left(\mathrm{Fe}^{3+}\right)$ & $.18-.51 \times 10^{6 b}$ & 245 & $5.0-41 \times 10^{-10 b}$ & 6.2 & 5.3 & 3.8 \\
\hline $18.0\left(\mathrm{Fe}^{3+}\right)$ & $.55-.88 \times 10^{6 b}$ & 502 & $4.6-12 \times 10^{-10 b}$ & 5.3 & 4.6 & 3.2 \\
\hline $42.0\left(\mathrm{Fe}^{3+} \alpha-\mathrm{CH}\right)$ & $2.2 \times 10^{6}$ & 690 & $1.1 \times 10^{-10}$ & 5.3 & 4.4 & 3.0 \\
\hline $13.7\left(\mathrm{Fe}^{2+} \beta-\mathrm{CH}_{2}\right)$ & $6.7 \times 10^{6}$ & 735 & $5.8 \times 10^{-11}$ & 4.3 & 3.4 & 2.3 \\
\hline $16.5\left(\mathrm{Fe}^{2+} \beta-\mathrm{CH}_{2}\right)$ & $8.5 \times 10^{6}$ & 502 & $2.5 \times 10^{-11}$ & $\geq 6.6^{d}$ & 4.1 & 3.1 \\
\hline $20.3\left(\mathrm{Fe}^{2+} \beta-\mathrm{CH}_{2}\right)$ & $10.9 \times 10^{6}$ & 860 & $2.5 \times 10^{-11 e}$ & $\geq 6.6^{d, e}$ & $3.5^{e}$ & $2.3^{e}$ \\
\hline
\end{tabular}

${ }^{a}$ Line position in ppm at $5^{\circ} \mathrm{C}$.

$b$ These resonances are not assigned. Range is due to uncertainty in diamagnetic contribution to line positions.

c Distances calculated after correcting for contribution from isotropic hyperfine interaction and a nonparamagnetic contribution of $95 \mathrm{rad} \cdot \mathrm{sec}^{-1}$.

${ }^{d}$ For this correlation time the dipolar contribution must be less than the uncertainty in estimating line widths, i.e., ca. $20 \mathrm{~Hz}$.

- This resonance corresponds to two protons. The contribution to the line width from nonequivalence is estimated from the difference in line width at $220 \mathrm{MHz}$ and $60 \mathrm{MHz}$ to be $\mathrm{ca} .95 \mathrm{rad} \cdot \mathrm{sec}^{-1}$.

resonances at the two frequencies is not possible because many of the resonances which are resolved at $220 \mathrm{MHz}$ collapse to singls lines at $60 \mathrm{MHz}$. However the resonance which occurs at $22 \mathrm{ppm}$ at $30^{\circ} \mathrm{C}$ is resolved as a single line at $60 \mathrm{MHz}$. This same resonance is resolved as a single line at 13.7 ppm in the $5^{\circ} \mathrm{C} / 220 \mathrm{MHz}$ spectrum presented by Poe et al. (5). The line width in both of these spectra is $80 \mathrm{~Hz}$. This comparison at two temperatures is justified by the observation that no changes in line widths are observed in our data over this

the range $14-51 \mathrm{kG}$. Without knowing the dominant mechanism for electron relaxation this assumption cannot be justified rigorously. However, because the rotational motion of the protein is slow $\left(\tau_{R}>10^{-8} \mathrm{sec}\right)$ the electron relaxation rate is determined most likely by internal molecular motions such as overdamped normal modes. For a protein we would expect that the frequency spectrum resulting from such motions and causing electron relaxation would extend to a sufficiently high frequency that $\omega_{s} \tau_{v} \ll 1$ in the range $14-15 \mathrm{kG}$, where $\tau_{v}$ is the correlation time for these internal motions. temperature range. The resonances which correspond to two protons appear to be slightly broader at $220 \mathrm{MHz}$, which probably reflects a slight nonequivalence of the protons. Any frequency dependence of the line width would result in narrower lines at the higher frequency. To within $15 \mathrm{~Hz}$ the line widths are equal at the two frequencies and we conclude that $\tau_{e}$ must lie outside the range between $1 \times 10^{-11}$ and $4 \times 10^{-13}$ sec. (3) Using a very concentrated sample of reduced spinach ferredoxin we have measured the epr line width at $230^{\circ} \mathrm{K}$; it is greater than $1000 \mathrm{G}$. A lower limit for $\tau_{e}$ can be estimated from this line width by assuming $T_{1}=T_{2}$; thus $\tau_{e}$ is at least $c a .6 \times 10^{-11} \mathrm{sec}$ at this temperature. At room temperature, this time can only be shorter. Thus the value for $\tau_{e}$ estimated from the $\mathrm{nmr}$ line widths is consistent with that estimated from the epr line width.

Given a value for $\tau_{e}$, the distances between the various protons and the paramagnetic center can be calculated, since the dipolar contribution can be determined. The various distances are summarized in Table I 
for various values of the electronic relaxation time. From CPK molecular models we find that the $\beta-\mathrm{CH}_{2}$ protons are within 2.6$4.3 \AA$ of the metal atom. The maximum separation of the $\alpha$ proton is about $5.5 \AA$ while it can contact the iron at its position of closest approach. Because of the difficulty of defining a van der Waals' radius for an open shell atom $\left(\mathrm{Fe}^{3+}\right)$ we estimate the distance of closest approach to be the sum of the covalent radius of the metal ion and van der Waals' radius of the $\mathrm{H}$ atom; this sum is $2.3 \AA$. From the CPK model we measure $2.4 \AA$. From Table I we see that a correlation time less than $4 \times 10^{-13}$ sce leads to distances substantially smaller than the values allowed by the molecular models and we thus conclude that $\tau_{e} \approx 1 \times 10^{-11} \mathrm{sec}, \mathrm{a}$ value which leads to reasonable values of $r$ for those resonances for which assignments can be made.

The line width of the $37 \mathrm{ppm}$ resonance in the oxidized protein cannot be used to determine distance because of an unknown contribution to the line width from nonequivalence of the eight protons. The data of Crespi (11) suggests that this nonequivalence may be appreciable. In oxidized ferredoxin from Synechococcus lividus Crespi observes a line width of $c a .15 \mathrm{kHz}$ for the $37 \mathrm{ppm}$ resonance at $220 \mathrm{MHz}$, whereas we observe a line width of only $3.7 \mathrm{kHz}$ for this resonance at $60 \mathrm{MHz}$. This nearly linear dependence on field of the line width, although observed on samples of ferredoxin from different sources, strongly suggests that nonequivalence among the protons is the principal determinant of the line width.

At $60 \mathrm{MHz}$ the resonance at $14.7 \mathrm{ppm}$ in the oxidized protein is observed only as a shoulder on the tail of the resonances from the diamagnetic region. Consequently we are unable to estimate the line width at
$60 \mathrm{MHz}$ and therefore unable to estimate $\tau_{\text {。 }}$ in the oxidized protein.

In principle the various distances can be used in conjunction with the geometrical model of the iron-sulfur complex to generate possible stereochemical configurations for the active site. However this approach should be reserved for that time when the line assignments and line width arguments are on more secure footing.

\section{ACKNOWLEDGMENTS}

We acknowledge valuable discussions with Drs. W. R. Dunham, D. Gill, L. Rimai and R. H. Sands. We are particularly grateful to Dr. H. Crespi for permission to quote his unpublished results.

\section{REFERENCES}

1. Brintzinger, H., Palmer, G., And SAnds, R. H. (1966) Proc. Nat. Acad. Sci. U. S. A. 55, 397 .

2. Dunham, W. R., Palmer, G., Sands, R. H., ANd Bearden, A. J. (1971) Biochem. Biophys. Acta 258, 373.

3. Gibson, J. F., Hall, D. O., Thornley, J. H. M., and Whatley, F. R. (1966) Proc. Nat. Acad. Sci.U.S.A. 56, 987.

4. Palmer, G., Dunham, W. R., Fee, J., Sands, R. H., Irzuka, T., and Yonetani, T. (1971) Biochem. Biophys. Acla 245, 201.

5. Poe, M., Phillips, W. D., Glickson, J. D., McDonald, C. C., and San Pietro, A. (1971) Proc. Nal. Acad. Sci. U.S.A.68, 68.

6. Poe, M., Phillips, W. D., McDonald, C. C., and Lovenberg, W. (1970) Proc. Nat. Acad. Sci. U.S.A. 65, 797.

7. Petering, D., and Palmer, G. (1970) Arch. Biochem. Biophys. 141, 456.

8. Eaton, D. R., Josey, A. D., And Benson, R. E. (1967) J. Amer. Chem. Soc. 89, 4040.

9. McDonald, C. C., and Phillips, W. D. (1969) J. Amer. Chem. Soc. 91, 1513.

10. Carrington, A., and McLachlan, A. D. (1967) Introduction to Magnetic Resonance, p. '228. Harper and Row.

11. Crespi, H. (1971) personal communication. 